nutrition a $9 \%$ branched-chain amino acid-free solution of amino acids reduced plasma leucine levels in acutely ill infants and young children with maple syrup urine disease. The steps in treatment included rapid correction of dehydration or acidosis; recommended dietary allowance of calories; age dependent minimal requirement of amino acids; supplements of isoleucine or valine to prevent deficiency; and treatment of hyperglycemia with insulin. The use of the modified parenteral nutrition was associated with relief from vomiting, improvement in consciousness, and return to the maple syrup urine disease formula. The authors stress the value of this therapy in all acutely ill patients with plasma leucine levels between 1 and 2 mol per liter who cannot tolerate enteral nutritional therapy. (Berry GT et al. Branched-chain amino acid-free parenteral nutrition in the treatment of acute metabolic decampensation in patients with maple syrup urine disease. Nㅡㅁ J Med Jan 17, 1991; 324:175-179).

COMMENT. Peritoneal dialysis and hemodialysis have proved effective in the treatment of acute metabolic decompensation in these patients but in those who are not camatose, this form of modified nutritional therapy may be more appropriate. The treatment was thought to lower the plasma leucine levels by favoring the flux of branched-chain amino acids into body protein.

\title{
NYSTAGMUS OF PELIZAEUS-MERZBACHER DISEASE
}

Magnetic search-coil oculography of three brothers, aged 20, 22, and 25, with Pelizaeus-Merzbacher disease (PND) was used to demonstrate nystagmus not obvious on inspection at the W. K. Kellogg Eye Center, University of Michigan, Ann Arbor, MI. The method disclosed the presence of binocular elliptical pendular nystagmus in two patients and upbeat nystagmus in all three patients. This combination of elliptical pendular and upbeat nystagmus is not described in any other childhood neurodegenerative disease. In conjunction with MRI findings and supportive clinical signs a diagnosis of $P M D$ is strongly indicated. (Trobe JD et al. Nystagmus of Pelizaeus-Merzbacher disease. A magnetic search-coil study. Arch Neurol Jan 1991; 48:87-91).

COMMENT. PMD may be classified by age of onset into a classical infantile type, congenital type, and adult onset type. The classical and congenital forms have $\mathrm{X}$-linked recessive inheritance whereas the adult onset type is autosomal dominant in inheritance. Nystagmus and head tremor are the first signs followed by spasticity, ataxia, choreoathetosis, optic atrophy, and dementia. PMD must be differentiated from congenital nystagmus or spasmus nutans, metachromatic leukodystrophy, adrenoleukodystrophy, Krabbe's disease, and Cockayne's syndrome. The MRI in PMD shows brain atrophy, increased T2 signal in white matter, and decreased signal in basal ganglia and thalami. Pathological correlations of upbeat nystagmus have shown dysmyelination of the pontomedullary tegmentum. 